\title{
Lipidomic analysis of serum samples from migraine patients
}

\author{
Caixia Ren', Jia Liü ${ }^{2}$ Juntuo Zhou ${ }^{2}$, Hui Liang ${ }^{2}$, Yayun Wang ${ }^{3}$, Yinping Sun ${ }^{4}$, Bin Ma ${ }^{3 *}$ and Yuxin Yin ${ }^{2,5^{*}}$
}

\begin{abstract}
Background: Migraine is a prevalent, disabling type of primary headache disorder associated with a high socioeconomic burden. The clinical management of migraine is challenging. This study was to identify potential serum lipidomic biomarkers of migraine.

Methods: The serum lipidomic profile of migraine sufferers was compared with healthy individuals using Liquid Chromatography coupled to Mass Spectrometry (LC-MS). Volcano plot analysis by Student's t-test was performed to identify the differential metabolites. Receiver operating characteristic (ROC) curves were constructed and the area under ROC curves (AUC) was calculated to evaluate whether the metabolites could be efficiently exploited for constructing a sensitive biomarker of migraine.

Results: A total of 29 serum metabolites from 4 classes of lipids including acylcarnitines, non-alpha-hydroxy-sphingosine ceramides (Cer_NSs), lysophosphatidylcholines (lysoPCs) and lysophosphatidylethanolamines (lysoPEs) were significantly different in migraine patients and controls. Of note, Cer_NSs were significantly elevated and lysoPEs were significantly decreased in migraine patients. LysoPE 18:1, lysoPE 18:2 and lysoPE 22:5 were found to be decreased in both positive and negative ion mode. Moreover, except for lysoPC 20:0, other lysoPCs were decreased in migraine patients. ROC curve analysis indicated that lysoPC 16:0 and lysOPC 20:0 are potential sensitive and specific biomarkers for migraine.
\end{abstract}

Conclusion: LysoPC 16:0 and lysoPC 20:0 may be potential biomarkers for migraine. We suggest therapeutic management of these metabolites may be helpful in the prevention and treatment of migraine.

Keywords: Migraine, Lipidomics, Biomarkers, LysoPC, Acylcarnitine, Cer_NS, LysoPE

\section{Background}

Migraine is a common headache disorder with a lifetime prevalence of $13 \%$ in men and $33 \%$ in women [1]. The clinical features of migraine include intense and pulsating head pain localized to one side of the head that can effectively disable its sufferers for up to $72 \mathrm{~h}$, Accompanying symptoms of migraine in addition to the headache include nausea, vomiting, and hypersensitivity to lights, sounds and/or smells, all of which can be aggravated by physical activity [2]. Migraine is the 8th most burdensome disease in the world overall, and the 4th most burdensome disease in women according to the 2012 Global Burden of Disease

\footnotetext{
* Correspondence: mabin0352@126.com; yinyuxin@bjmu.edu.cn

${ }^{3}$ Department of Neurology, Dongzhimen Hospital Affiliated to Beijing University of Chinese Medicine, Beijing 100700, China

${ }^{2}$ Institute of Systems Biomedicine, Department of Pathology, School of Basic Medical Sciences, Peking University Health Science Center, Beijing 100191, China

Full list of author information is available at the end of the article
}

study, as it is associated with poor health-related quality of life, missed work days, and a high economic burden [3]. Despite intensive research, the exact pathologic mechanism of migraine is still poorly understood, making the discovery of more effective treatments extremely difficult. Many medications are used to treat migraine, however, none of these pharmaceutical agents has shown universal treatment efficiency, and side effects often limit their use [4]. At the same time, recurrences of symptoms may require increased use of a given drug, which may lead to excessive use and dissatisfaction with available treatments [5]. Therefore, seeking for new potential biomarkers is very important to help prevent and treat migraine.

In addition to genomics and proteomics, lipidomics have been employed as a metabolomic approach for investigation of the qualitative and quantitative profiles of lipid metabolites from serum, plasma, tissue, cells and organisms [6]. Circulating lipid metabolites are associated with many 
physiologic and pathologic processes in the body, and may therefore be of use for selecting biomarkers for prevention and treatment of disease [7]. To the best of our knowledge, there are few reports on lipidomic profiling of migraine.

In this study, we aimed to examine serum lipidomic metabolites with Liquid Chromatography coupled to Mass Spectrometry (LC-MS) to identify potential migraine biomarkers which may provide useful fundamental information for the prevention and treatment of migraine.

\section{Methods}

\section{Patients}

Twenty patients with migraine without aura, and 20 healthy control subjects in whom migraine was excluded by examination participated in this study (Table 1). The control subjects were recruited from hospital and laboratory personnel, and were age and sex matched with the study patients. Migraine was diagnosed by physicians specialized in this area of medicine utilizing the current International Headache Society (IHS) criteria (ICHD-3, beta version currently available at https://www.ichd3.org/) [2]. Subjects with hypertension, diabetes, high cholesterol, morbid obesity, history of cardiovascular events, chronic renal failure, liver cirrhosis, thyroid diseases, history of significant head trauma, malnutrition, pregnancy, cigarette smoking, alcohol and substance abuse, chronic neurologic illnesses, including epilepsy, Parkinson's disease, Huntington's disease, Alzheimer's disease, Wilson's disease were excluded from both groups. None of these patients were taking any medication except for treatment of migraine attacks. On the day of study, patients were all headache free for at least 5 days. These patients abstained from taking any medication until the end of the study period. All subjects gave informed consent for inclusion before they participated in the study. The study was conducted in accordance with the Declaration of Helsinki, and the protocol was approved by the Ethics Committee of the Beijing University of Chinese Medicine (ECPJ-BDY-2016-06) (Additional file 1: Figure S1).

\section{Chemicals and reagents}

Formic acid, HPLC grade methanol, acetonitrile (ACN) and isopropanol (IPA) were obtained from Fisher Scientific. Chloroform was obtained from Tong Guang Fine Chemicals Company (Beijing, China). Free fatty acid (FFA) 19:0 and ammonium acetate was purchased from Sigma-Aldrich

Table 1 Baseline characteristics of the study populations (mean \pm SD)

\begin{tabular}{lll}
\hline Characteristics & Control & Migraine \\
\hline Numbers & 20 & 20 \\
Gender (Male/Female) & $8 / 12$ & $8 / 12$ \\
Age (year) & $32.85 \pm 7.47$ & $31.85 \pm 9.1$ \\
\hline
\end{tabular}

(St. Louis, MO, USA). Ultra-pure water was supplied by a Millipore system (Millipore, Billerica, MA, USA).

\section{Sample preparations}

Lipids were extracted from serum samples by a modified Folch method [8]. Typically, $100 \mu \mathrm{L}$ of serum were aliquoted into a $0.6 \mathrm{ml}$ Eppendorf tube and mixed with $400 \mu \mathrm{L}$ of chloroform/methanol $(2: 1, V / \mathrm{V})$ containing $20 \mu \mathrm{g} / \mathrm{ml}$ of free fatty acid 19:0 as an internal standard. After vortexing for $10 \mathrm{~min}$, the mixture was centrifuged at $13000 \mathrm{rpm}$ at $4{ }^{\circ} \mathrm{C}$ for $20 \mathrm{~min}$. The lower lipid containing chloroform phase was evaporated with a speed vacuum, and the residue was stored at $-80{ }^{\circ} \mathrm{C}$ for further analysis. All samples were processed in the same laboratory to avoid bias.

\section{High-performance liquid chromatography}

An Ultimate 3000 ultra high performance liquid chromatography (UHPLC) system coupled to Q-Exactive MS (Thermo Scientific) was used for lipid separation and detection. Samples were reconstituted in $20 \mu \mathrm{L}$ chloroform/methanol $(1: 1, V / \mathrm{V})$ and diluted three times in IPA/ACN/water (2:1:1, V/V/V). After centrifugation at $12000 \mathrm{rpm}$ for $15 \mathrm{~min}, 5 \mu \mathrm{L}$ of supernatant were injected for LC-MS/MS analysis.

Chromatographic separation was performed on a reversed phase X select CSH C18 column $(4.6 \mathrm{~mm} \times 100 \mathrm{~mm}$, $2.5 \mu \mathrm{m}$, Waters, USA), which is consistent with what has been reported [9]. Two solvents were used for gradient elution: (A) ACN/water (3:2, V/V), (B) IPA/ACN (9:1, V/V). Both $\mathrm{A}$ and $\mathrm{B}$ contained $10 \mathrm{mM}$. ammonium acetate and $0.1 \%$ formic acid. The gradient program was: 0 min- $40 \% \mathrm{~B}$; 2 min-43\% B; $2.1 \min -50 \%$ B; $12 \min 60 \%$ B; $12.1-75 \%$ B; 18 min-99\% B; 19 min-99\% B; 20 min-40\% B; 25 min-40\% B. The column temperature was maintained at $50{ }^{\circ} \mathrm{C}$, and the flow rate was set to $0.6 \mathrm{ml} / \mathrm{min}$.

\section{Mass spectrometry}

Mass spectrometric detection was performed by electrospray ionization in both positive ion mode and negative ion mode. The source voltage was maintained at $3.3 \mathrm{kV}$ in the positive ion mode and $2.8 \mathrm{kV}$ in the negative ion mode. All other interface settings were identical for both positive ion mode and negative ion mode. The capillary temperature, sheath gas flow and auxiliary gas flow were set at $320{ }^{\circ} \mathrm{C}, 40$ arb and 10 arb respectively. Data were collected in a data-dependent top 10 scan mode. Survey full-scan MS spectra (mass range $m / z 80$ to 1200) were acquired with resolution $R=70,000$ and AGC target 1e6. MS/MS fragmentation was performed using high-energy c-trap dissociation (HCD) with resolution $R=17,500$ and AGC target 1e5. The stepped normalized collision energy (NCE) was set to 15,30 , and 45 respectively. 
External mass calibration was applied before every sequence run.

The stability of retention time, mass accuracy and intensity is essential in LC-MS based lipidomic analysis. A pooled serum sample was therefore prepared as QC to assess the stability of the instrument and ensure the reliability of the data. A QC sample was run before and after the sequence and in every 10 sample runs in the sequence in order to ensure the reproducibility of the data.

\section{Data processing and analysis}

The acquired raw data were processed using MSDIAL according to the instructions in the software tutorial [10]. Datasets containing $\mathrm{m} / \mathrm{z}$ values, retention time, and peak area were exported as an Excel file, and then the Excel file was imported into the Metabo Analyst 3.0 Web service [11] for multivariate analysis. Principal component analysis (PCA) which is an unsupervised chemometric method, was used to obtain an overall picture of the whole data sets, and to see if there were any clustering, trends, or outliers. Orthogonal partial least squares-discriminant analysis (orthoPLS-DA) was used for further data analysis. Volcano plot analysis with foldchange $(\mathrm{FC})>1.5$ and false discovery rate $(\mathrm{FDR})<0.05$ by Student's t-test was performed to identify the differential metabolites. ROC curves were constructed and the area under ROC curves (AUC) was calculated to investigate whether the characteristics of the metabolites that differed significantly between two groups could be efficiently exploited for constructing a sensitive biomarker of migraine status.

\section{Results}

\section{Patient characteristics}

A total of 20 migraine patients and 20 controls were recruited for screening and identification of promising metabolites (Table 1). The mean ages for migraine patients and controls were $31.85 \pm 9.1$ and $32.85 \pm 7.47$ years, respectively $(p=0.819)$.

The causes of migraine were showed in Table 2. Most of them (16/20) suffered from anxiety and insomnia; 2

Table 2 Causes of migraine in this study

\begin{tabular}{ll}
\hline Causes of migraine & Numbers \\
\hline Anxiety and insomnia alone & 6 \\
Anxiety and insomnia accompanied by: & 2 \\
Physical stimuli such as light or smell & 4 \\
Dietary factors such as cold drink or coffee & 2 \\
Physical activities such as running or taking bus & 2 \\
Weather changes such as wind or cold stimuli & 4 \\
Physical stimuli such as light, noise or smell alone & 20 \\
Total &
\end{tabular}

of 16 cases were accompanied by physical stimuli such as light or smell; 4 of 16 were accompanied by dietary factors such as cold drink or coffee; 2 of 16 were accompanied by physical activities such as running or taking bus; and 2 of 16 were accompanied by weather changes such as wind or cold stimuli. The remaining 4 (4/20) migraine were resulted from physical stimuli such as light, noise or smell alone.

\section{Identification of potential metabolic biomarkers}

PCA score plots which include migraine patients, healthy controls and quality control (QC) samples are shown in Fig. 1a (positive ion mode) and 1D (negative ion mode). All samples scattered in these figures fell into the 95\% confidence interval. QC samples (blue) clustered together tightly, reflecting the stability of the LC-MS system and showing that the quality of all the LC-MS data for this study were satisfactory. The migraine group (M group, green) and healthy control group (C group, pink) were clustered together and separated from each other, despite a few overlapping data points in these two groups. This suggested that the involved metabolites were perturbed in the migraine group. In order to maximize the separation of the migraine and control groups, we found it was necessary to input a priori information about these two classes and use supervised models, such as Orthogonal partial least squares-discriminant analysis (orthoPLS-DA). The score plot in the orthoPLS-DA showed better clustering tendency between the migraine (green) and control groups (pink) as shown in Fig. 1b (positive ion mode) and Fig. 1e (negative ion mode). The percentage of extracted variance related to class information was $13 \%$ (positive ion mode) and 12\% (negative ion mode) and explained $73.2 \%$ of the variance of class variables in positive ion mode $\left(R^{2} Y=0.732\right)$ (Fig. 1c) and $74.6 \%$ of the variance of class variables in negative ion mode $\left(R^{2} Y=0.746\right)$ (Fig. 1f). These multivariate analyses showed significant metabolic differences in the migraine and control groups.

A total of 1026 metabolites in positive ion mode and 923 metabolites in negative ion mode were evaluated in these two groups. After quality control analysis and further volcano plot analysis with $\mathrm{FC}>1.5$ and FDR $<0.05$ using the Student's t-test, 29 lipidomic metabolites from 4 lipid classes including acylcarnitines, non-alpha-hydroxy-sphingosine ceramides (Cer_NSs), lysophosphatidylcholines (lysoPCs), and lysophosphatidylethanolamines (lysoPEs) were significantly different in migraine patients and controls. Seven of 29 metabolites showed significantly higher levels in migraine patients and 22 exhibited lower levels (Table 3). Of note, Cer_NSs were significantly elevated in migraine patients, and lysoPEs were significantly decreased in migraine patients. Moreover, decreased lysoPE 18:1, lysoPE 18:2 and lysoPE 22:5 were detected in both positive ion mode and negative ion mode. Box plots showed similar results for 


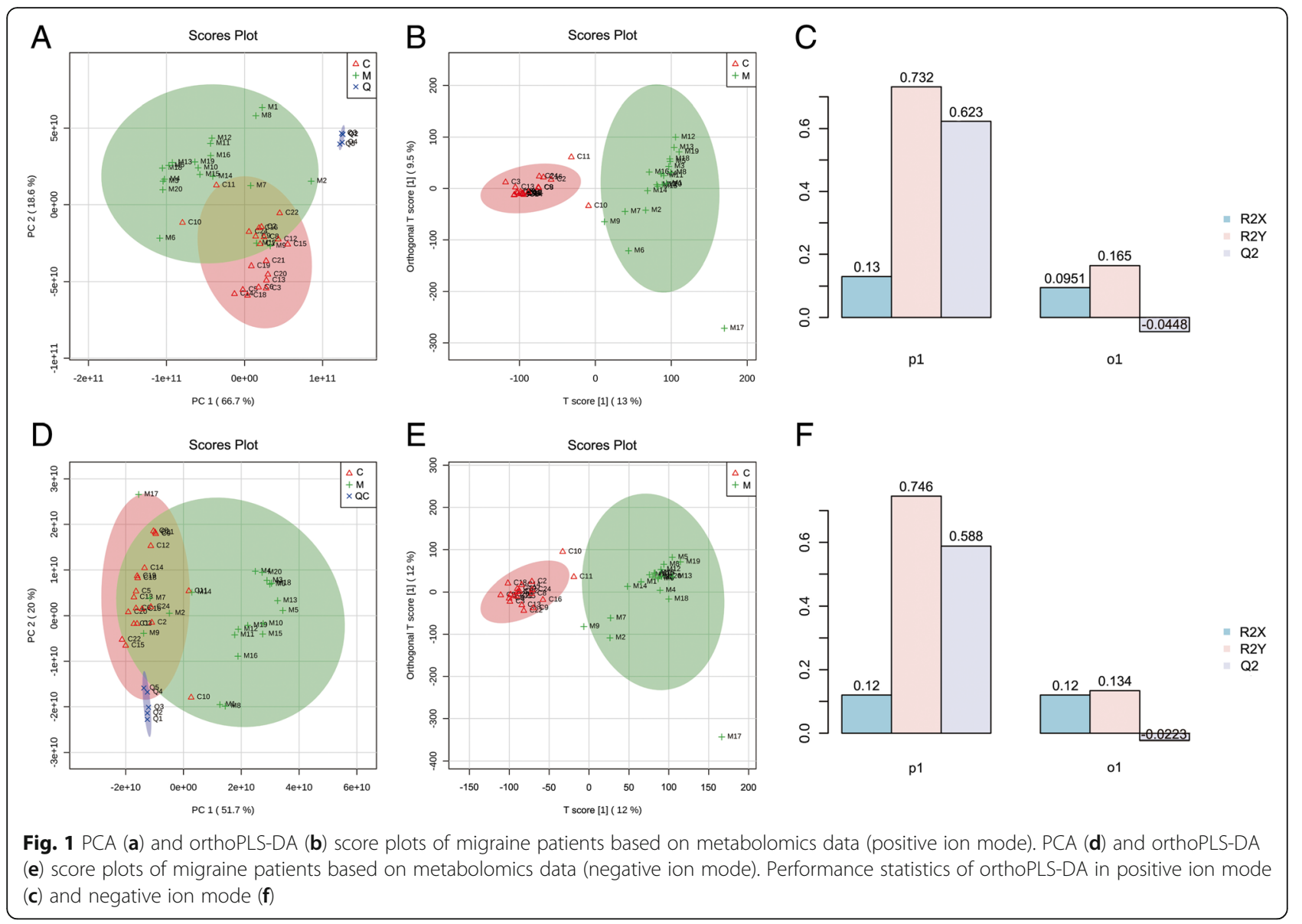

these lysoPEs in both positive and negative ion mode (Fig. 2). Additionally, except for lysoPC 20:0, other lysoPCs decreased in migraine patients.

\section{Diagnostic performance of metabolites}

A receiver operating characteristic (ROC) curve analysis of potential biomarker levels for differentiating migraine patients is shown in Table 3. The optimal cutoff points as calculated with Youden's index, sensitivities, specificities, and AUC values are listed. Of these 29 metabolites, 16 showed AUC $>0.80$ when migraine patients and controls were compared.

The AUC value of lysoPC 16:0 was 0.9 (95\% CI = 0.7820.986), which was the highest of these AUC values, indicating a sensitivity of $90 \%$ and specificity of $80 \%$ for differentiation of migraine patients and controls. The AUC value of lysoPC 20:0 was 0.895 (95\% CI =0.731-0.986), indicating a sensitivity of $90 \%$ and specificity of $90 \%$ in these two groups (Fig. 3). LysoPE 18:2, LysoPE 18:1, and lysoPC 22:5 were detected both in positive and negative ion modes, and the AUC values were 0.845 (90\% sensitivity and $80 \%$ specificity), 0.835 (80\% sensitivity and 70\% specificity) and 0.802 (80\% sensitivity and $80 \%$ specificity) respectively (Table 4 ).

\section{Discussion}

This study examined the lipidomic profiles of migraine patients based on LC-MS. We identified 29 metabolites from 4 lipid classes that were significantly different in migraine patients and controls.

\section{Acylcarnitine}

Acylcarnitines are intermediates in fatty acid and amino acid breakdown generated from the conversion of acylCoA species by the action of carnitine palmitoyltransferase 1 (CPT1) [12]. After production of acylcarnitines by CPT1, the mitochondrial inner membrane transporter carnitine acylcarnitine translocase (CACT, or SLC25A20) transports the acylcarnitines into the mitochondrial matrix. Finally, the enzyme CPT2 reconverts acylcarnitines back into free carnitine and long-chain acyl-CoAs [13]. The formation of carnitine conjugates is crucial for the transport of activated fatty acids (acyl-CoAs) from the cytosol across the inner mitochondrial membrane into the matrix where fatty acid oxidation takes place. It has now become clear that a net efflux of acylcarnitine species from the mitochondria into the cytosol and ultimately into plasma is particularly important in situations of impaired fatty acid oxidation for prevention of accumulation of potentially toxic acyl-CoA 
Table 3 Identification of potential serum lipidomic biomarkers in migraine patients

\begin{tabular}{|c|c|c|c|c|c|c|}
\hline \multirow{2}{*}{\multicolumn{7}{|c|}{ Ion adduct Retention time(min) Experimental Mass,M/z Fold change (Migraine/Control) $p$ value }} \\
\hline & & & & & & \\
\hline 1 & Acylcarnitine15:3 & {$[\mathrm{M}]+$} & 2.22 & 380.2813 & $3.839 \uparrow$ & 0.005 \\
\hline 2 & Acylcarnitine 21:4 & {$[\mathrm{M}]+$} & 3.37 & 462.3571 & $3.520 \uparrow$ & 0.000 \\
\hline 3 & Acylcarnitine 11:1 & {$[\mathrm{M}]+$} & 1.95 & 328.248 & $2.094 \uparrow$ & 0.004 \\
\hline 4 & Acylcarnitine 14:3 & {$[M]+$} & 2.01 & 366.2634 & $0.478 \downarrow$ & 0.007 \\
\hline 5 & Acylcarnitine 10:1 & {$[M]+$} & 1.9 & 314.2327 & $0.465 \downarrow$ & 0.001 \\
\hline 6 & Acylcarnitine 24:0 & {$[M]+$} & 9.93 & 512.4677 & $0.435 \downarrow$ & 0.009 \\
\hline 7 & Acylcarnitine 10:0 & {$[M]+$} & 1.95 & 316.2485 & $0.402 \downarrow$ & 0.002 \\
\hline 8 & Acylcarnitine 26:0 & {$[\mathrm{M}]+$} & 12.49 & 540.498 & $0.377 \downarrow$ & 0.000 \\
\hline 9 & Acylcarnitine 20:0 & {$[M]+$} & 6.09 & 456.4047 & $0.348 \downarrow$ & 0.000 \\
\hline \multicolumn{7}{|c|}{ Cer_NS (positive ion) } \\
\hline 10 & $\begin{array}{l}\text { Cer_NS 34:1; } \\
\text { Cer_NS(d18:1/16:0) }\end{array}$ & {$[\mathrm{M}+\mathrm{H}]+$} & 14.75 & 538.5197 & $3.520 \uparrow$ & 0.000 \\
\hline 11 & $\begin{array}{l}\text { Cer_NS 36:3; } \\
\text { Cer_NS(d18:2/18:1) }\end{array}$ & {$[\mathrm{M}+\mathrm{H}]+$} & 13.19 & 562.5194 & $2.723 \uparrow$ & 0.000 \\
\hline 12 & $\begin{array}{l}\text { Cer_NS 36:2; } \\
\text { Cer_NS(d18:2/18:0) }\end{array}$ & {$[\mathrm{M}+\mathrm{H}]+$} & 14.86 & 564.5356 & $2.430 \uparrow$ & 0.000 \\
\hline \multicolumn{7}{|c|}{ lysoPC (positive ion) } \\
\hline 13 & IysoPC 20:0; PC(20:0/0:0) & {$[\mathrm{M}+\mathrm{H}]+$} & 5.11 & 552.4031 & $2.860 \uparrow$ & 0.000 \\
\hline 14 & IysoPC 22:5; PC(22:5(4Z,7Z,10Z,13Z,16Z)/0:0) & {$[\mathrm{M}+\mathrm{H}]+$} & 3.06 & 570.3521 & $0.474 \downarrow$ & 0.002 \\
\hline 15 & lysoPC 22:0; PC(22:0/0:0) & {$[\mathrm{M}+\mathrm{H}]+$} & 8.07 & 580.4342 & $0.452 \downarrow$ & 0.012 \\
\hline 16 & lysoPC 18:2; PC(18:2(6Z,9Z)/0:0) & {$[\mathrm{M}+\mathrm{H}]+$} & 3.09 & 520.3403 & $0.430 \downarrow$ & 0.019 \\
\hline 17 & lysoPC 15:0; PC(15:0/0:0) & {$[\mathrm{M}+\mathrm{H}]+$} & 3.14 & 482.3243 & $0.423 \downarrow$ & 0.000 \\
\hline 18 & lysoPC 14:0; PC(14:0/0:0) & {$[\mathrm{M}+\mathrm{H}]+$} & 2.89 & 468.309 & $0.393 \downarrow$ & 0.000 \\
\hline 19 & lysoPC 24:0; PC(24:0/0:0) & {$[\mathrm{M}+\mathrm{H}]+$} & 10.33 & 608.475 & $0.337 \downarrow$ & 0.000 \\
\hline 20 & lysoPC 16:0; PC(16:0/0:0) & {$[\mathrm{M}+\mathrm{H}]+$} & 5.22 & 496.3397 & $0.244 \downarrow$ & 0.001 \\
\hline \multicolumn{7}{|c|}{ lysoPE (positive ion) } \\
\hline 21 & lysoPE 18:2; PE(18:2(6Z,9Z)/0:0) & {$[\mathrm{M}+\mathrm{H}]+$} & 3.23 & 478.2933 & $0.466 \downarrow$ & 0.015 \\
\hline 22 & lysoPE 18:1; PE(18:1(17Z)/0:0) & {$[\mathrm{M}+\mathrm{H}]+$} & 3.98 & 480.3085 & $0.461 \downarrow$ & 0.023 \\
\hline 23 & lysoPE 22:5; PE(22:5(4Z,7Z,10Z,13Z,16Z)/0:0) & {$[\mathrm{M}+\mathrm{H}]+$} & 3.05 & 528.2969 & $0.411 \downarrow$ & 0.007 \\
\hline 24 & lysoPE 24:0; PE(24:0/0:0) & {$[\mathrm{M}+\mathrm{H}]+$} & 10.62 & 566.4199 & $0.349 \downarrow$ & 0.016 \\
\hline \multicolumn{7}{|c|}{ lysoPE (negative ion) } \\
\hline \multirow[t]{4}{*}{25} & IysoPE 20:3; PE(20:3(5Z,8Z,11Z)/0:0) & {$[\mathrm{M}-\mathrm{H}]-$} & 3.73 & 502.2946 & $0.426 \downarrow$ & 0.014 \\
\hline & lysoPE 18:1; PE(18:1(17Z)/0:0) & {$[\mathrm{M}-\mathrm{H}]-$} & 4.02 & 478.2952 & $0.401 \downarrow$ & 0.014 \\
\hline & lysoPE 18:2; PE(18:2(6Z,9Z)/0:0) & {$[\mathrm{M}-\mathrm{H}]-$} & 3.26 & 476.2791 & $0.346 \downarrow$ & 0.007 \\
\hline & lysoPE 22:5; PE(22:5(4Z,7Z,10Z,13Z,16Z)/0:0) & {$[\mathrm{M}-\mathrm{H}]-$} & 3.27 & 526.2956 & $0.388 \downarrow$ & 0.014 \\
\hline 26 & IysoPE 18:3; PE(18:3(6Z,9Z,12Z)/0:0) & {$[\mathrm{M}-\mathrm{H}]-$} & 2.89 & 474.2632 & $0.380 \downarrow$ & 0.002 \\
\hline 27 & $\begin{array}{l}\text { lysoPE 22:6; PE(22:6(4Z,7Z,10Z,13Z,16Z,19Z)/ } \\
\text { 0:0) }\end{array}$ & {$[\mathrm{M}-\mathrm{H}]-$} & 3.12 & 524.2782 & $0.338 \downarrow$ & 0.003 \\
\hline 28 & IysoPE 20:4; PE(20:4(5Z,8Z,11Z,14Z)/0:0) & {$[\mathrm{M}-\mathrm{H}]-$} & 3.29 & 500.2796 & $0.332 \downarrow$ & 0.003 \\
\hline 29 & IysoPE 22:4; PE(22:4(7Z,10Z,13Z,16Z)/0:0) & {$[\mathrm{M}-\mathrm{H}]-$} & 3.9 & 528.311 & $0.309 \downarrow$ & 0.014 \\
\hline
\end{tabular}

intermediates in the mitochondrion [9]. Changes in plasma and/or urinary acylcarnitine profiles are used to detect disorders in fatty acid and amino acid oxidation [14, 15]. A broad spectrum of short-, medium-, long- and very long- chain acylcarnitine species is generated from multiple metabolic routes [16].

The current study showed 3 elevated odd-numbered (acylcarnitine 15:3, 21:4, 11:1) and 6 decreased even- 

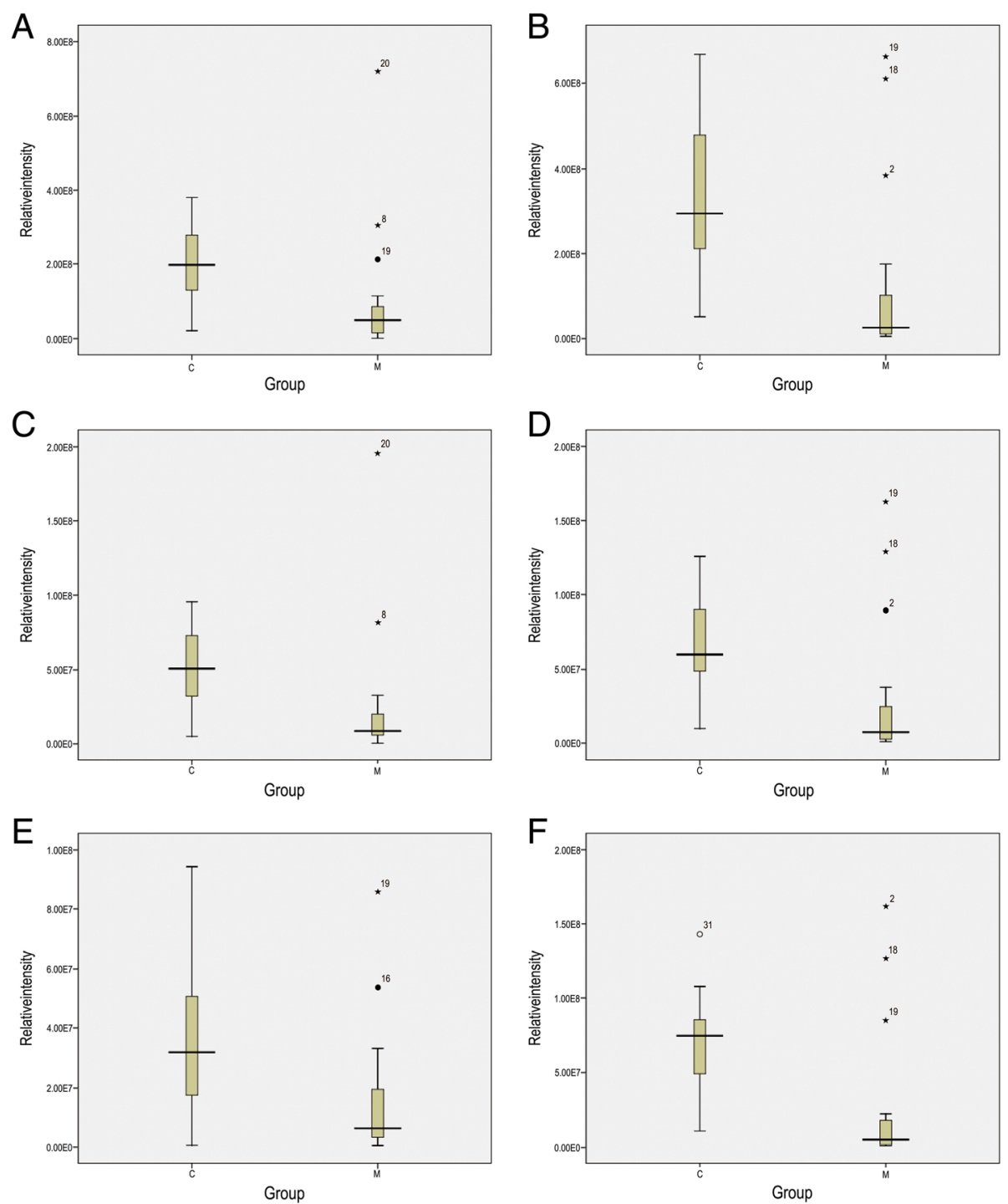

Fig. 2 Boxplots representing relative abundance of: (a) lysoPE 18:2, [M+H]+; (B) lysoPE. 18:1, [M + H]+; (C) lysoPE22:5, [M + H]+; (D) lysoPE 18:2, $[\mathrm{M}-\mathrm{H}]-;$ (E) lysoPE 18:1, [M-H]-; (F) lysoPE22:5, [M-H]-

numbered forms $(14: 3,10: 1,24: 0,10: 0,26: 0,20: 0)$ of medium- to very long-chain acylcarnitines (C10-C26) are found in migraine patients as compared to controls, indicating CPT1 activity for even-numbered acylcarnitine and CPT2 activity for odd-numbered acylcarnitine may be insufficient in these migraine patients. The exact mechanism through which even-numbered acylcarnitine or odd-numbered acylcarnitine might affect migraine remains unknown, and further studies may help determine the role of these acylcarnitines in migraine.

\section{Cer_NS}

Ceramides which are one of the simplest classes of sphingolipids, are lipid signaling molecules which regulate a broad range of both positive and negative cellular functions, including growth, proliferation, motility, adhesion, differentiation, senescence, apoptosis and autophagy [17-20]. Ceramides may be cytotoxic and activate pro-inflammatory cytokines, which promotes oxidative stress and endoplasmic reticulum (ER) stress [21]. Cytotoxic ceramides originating from the liver (or other organs/tissues) which enter the peripheral blood can cross the blood-brain barrier and exert neurotoxic and neurodegenerative effects in the brain [22]. Ceramides are associated with the pathogenesis of Alzheimer's disease (AD) [20]. Increased plasma levels of very longchain saturated ceramides (C22:0 and C24:0) were found to be predictive of memory loss and hippocampal atrophy in patients with cognitive impairment [23]. Inflammatory processes are often associated with migraine [24], and 

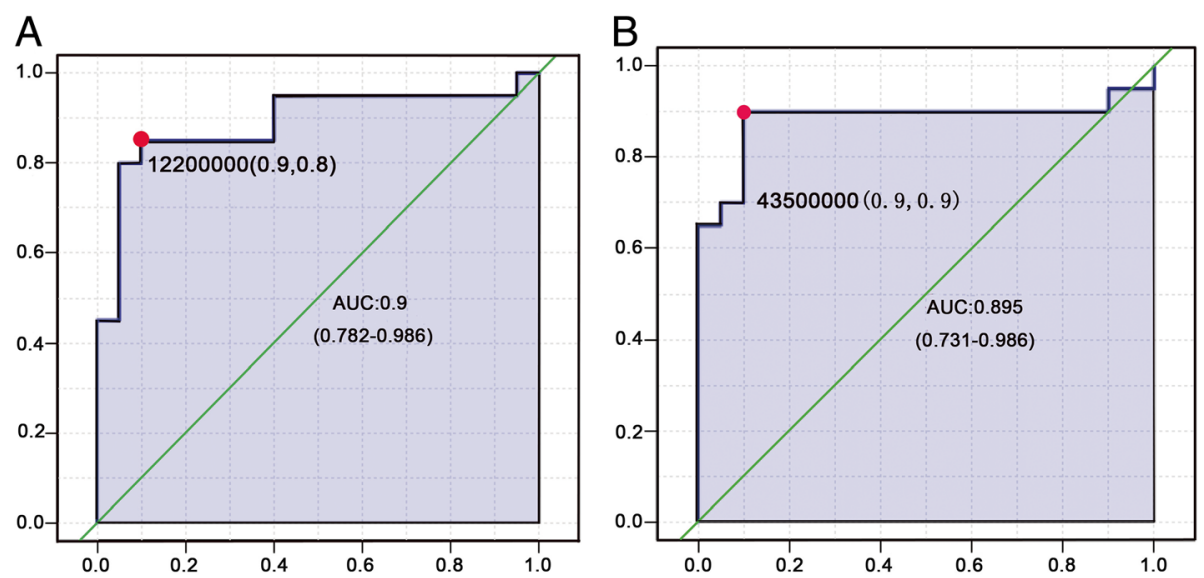

Fig. 3 ROC curves for (a) lysoPC 16:0; (b) lysoPC 20:0 using AUC $\geq 0.9$ with a 95\% confidence interval for comparing migraine and control groups

high levels of unsaturated very long-chain ceramides (Cer_NS 34:1, Cer_NS 36:3 and Cer_NS 36:2) in these patients may cause activation of pro-inflammatory cytokines, which consequently promote oxidative stress or ER stress and lead to tissue injury. Whether these very long-chain ceramides impair memory or bring about hippocampal injury remains unknown, and further work is needed to clarify the role of these ceramides in migraine.

\section{LysoPC and LysoPE}

LysoPC is a class of phospholipids derived from phosphatidylcholine $(\mathrm{PC})$ which is structural component of cell membranes. LysoPCs are produced by two pathways. The first is the result of partial hydrolysis of PC, so that one of the fatty acids is removed by the action of phospholipase A2 [25]. A second pathway for LysoPC formation occurs by the transfer of one fatty acid of PC to cholesterol by lecithin-cholesterol acyltransferase (LCAT), which is an enzyme that converts free cholesterol into cholesteryl ester. Cholesteryl ester is a more hydrophobic form of cholesterol that is sequestered in the core of lipoprotein particles and in the liver [26]. PE undergoes three successive methylation reactions by $\mathrm{PE}$ $\mathrm{N}$-methyl transferase (PEMT) for its full conversion to PC [27].

To date, the relationship between the serum levels of LysoPCs and migraine has not been described. This study showed up-regulation of lysoPC (20:0), and down-

Table 4 The AUC, 95\% confidence intervals (95\% CI), and sensitivity and specificity for ROC curves calculated at the optimal cutoff, together with the $p$-values for 16 significant lipidomic metabolites

\begin{tabular}{|c|c|c|c|c|c|c|c|}
\hline No. & Significant Metabolites & AUC & $95 \% \mathrm{Cl}$ & Sensitivity & Specificity & Cutoff value & $p$ value \\
\hline 1 & lysoPC 16:0 & 0.9 & $0.782-0.986$ & 0.9 & 0.8 & $1.22 \mathrm{e}+07$ & 0.000 \\
\hline 2 & lysoPC 20:0 & 0.895 & $0.731-0.986$ & 0.9 & 0.9 & $4.35 e+07$ & 0.000 \\
\hline 3 & Acylcarnitine 21:4 & 0.888 & $0.746-0.978$ & 0.8 & 0.8 & $1.35 e+08$ & 0.000 \\
\hline 4 & Cer_NS 34:1 & 0.88 & $0.721-0.965$ & 0.8 & 0.8 & $1.97 e+06$ & 0.000 \\
\hline 5 & lysoPE 24:0 & 0.86 & $0.695-0.96$ & 0.8 & 0.8 & $7.13 e+06$ & 0.000 \\
\hline 6 & lysoPC 14:0 & 0.86 & $0.718-0.949$ & 0.9 & 0.7 & $3.56 e+08$ & 0.000 \\
\hline 7 & Acylcarnitine $26: 0$ & 0.85 & $0.71-0.954$ & 0.8 & 0.9 & $3.84 e+07$ & 0.000 \\
\hline 8 & lysoPC 24:0 & 0.85 & $0.685-0.976$ & 0.8 & 0.9 & $7.27 e+06$ & 0.000 \\
\hline 9 & lysoPE $18: 2$ & 0.845 & $0.69-0.958$ & 0.9 & 0.8 & $1.18 \mathrm{e}+08$ & 0.000 \\
\hline 10 & lysoPE 18:1 & 0.835 & $0.691-0.945$ & 0.8 & 0.7 & $4.48 e+07$ & 0.000 \\
\hline 11 & lysoPC 22:0 & 0.83 & $0.686-0.95$ & 0.8 & 0.8 & $1.35 e+07$ & 0.000 \\
\hline 12 & lysoPC 15:0 & 0.828 & $0.655-0.949$ & 0.8 & 0.8 & $3.27 e+06$ & 0.000 \\
\hline 13 & Cer_NS 36:2 & 0.82 & $0.672-0.941$ & 0.8 & 0.8 & $6.36 e+06$ & 0.000 \\
\hline 14 & Acylcarnitine 15: 3 & 0.82 & $0.674-0.951$ & 0.8 & 0.8 & $7.42 e+06$ & 0.005 \\
\hline 15 & Acylcarnitine 20: 0 & 0.805 & $0.635-0.942$ & 0.8 & 0.8 & $6.39 e+06$ & 0.000 \\
\hline 16 & lysoPC 22:5 & 0.802 & $0.638-0.929$ & 0.8 & 0.8 & $1.5 e+08$ & 0.001 \\
\hline
\end{tabular}


regulation of lysoPCs (16:0, 24:0,14:0, 15:0, 22:0, 18:2, 22:5) in migraine patients. The AUC value of lysoPC 20:0 was 0.895 ( $90 \%$ sensitivity and $90 \%$ specificity), and the AUC value of lysoPC 16:0 was 0.9 (90\% sensitivity and $80 \%$ specificity), indicating lysoPC $16: 0$ and lysoPC 20:0 are potential sensitive and specific biomarkers for migraine. The reason these LysoPCs are altered in migraine is unclear. Oxidative stress, which arises because of an imbalance between the production of reactive oxygen species (ROS) and elimination by antioxidant defense mechanisms has been implicated in migraine [28-31]. Endogenous ROS can cause oxidative damage to DNA, lipids, proteins and extracellular matrix components, including proteoglycans and collagens. Oxidants may also confer susceptibility to other pathogenic processes by disrupting the functions of cytoprotective proteins, such as metabolic enzymes and cell membrane transporters [32]. In this study, most migraine patients suffered from anxiety and insomnia with or without other stimuli. These causes of migraine can lead to oxidative stress. When oxidative stress occurs, the generation of free radicals can activate the phospholipase A2, which hydrolyses PC (PE) to lysoPC (lysoPE). However, at the same time the oxidants disrupt the function of metabolic enzymes such as phospholipase A2, and result in the alterations of lysoPCs and lysoPEs. This mechanism requires further investigation.

LysoPE which is a lyso-type phospholipid, is a metabolic product of phosphatidylethanolamine (PE, a minor constituent of cell membranes) generated by phospholipase A2 [33]. LysoPE has been shown to mobilize intracellular $\mathrm{Ca}^{2+}$ through G-protein-coupled receptor (GPCR) in some cells types, such as MDA-MB-231 breast cancer cells, skov3 human ovarian cancer cells and human $\mathrm{SH}-$ SY5Y neuroblastoma cells [34-36]. Abnormal synaptic $\mathrm{Ca} 2+$ homeostasis and morphology may contribute to chronic neurodegenerative changes in migraine mice [37]. The decreased lysoPEs (18:1, 18:2, 22:5, 18:3, 22:6, 22:4, 20:4, 20:3) maybe lead to abnormal synaptic $\mathrm{Ca}^{2+}$ in migraine patients. Exogenous supplementation of these decreased lysoPEs maybe mobilize synaptic $\mathrm{Ca}^{2+}$ and be helpful for improving the prevention and treatment of migraine.

\section{Conclusions}

We determined 29 metabolites from 4 lipid classes were significantly different in migraine patients compared to controls: Acylcarnitine, Cer_NS, LysoPC and LysoPE. ROC curve analysis indicated that lysoPC 16:0 and lysoPC 20:0 may be new potential sensitive and specific biomarkers for migraine. We suggest that management of these metabolites may be of benefit in the prevention and treatment of migraine. Further studies should be performed in larger populations of subjects to validate the conclusions of this study.

\section{Additional file}

Additional file 1: The ethics of samples involved in the study. (JPEG $2444 \mathrm{~kb}$ )

\section{Abbreviations}

Cer_NS: Non-alpha-hydroxy-sphingosine ceramide; LysoPC: Lysophosphatidylcholine; LysoPE: Lysophosphatidylethanolamine; orthoPLS-DA: Orthogonal partial least squares-discriminant analysis; PCA: Principal component analysis; ROC: Receiver operating characteristic

\section{Acknowledgments}

Not applicable.

\section{Fundings}

This work was supported by grants to Yuxin Yin from the National Natural Science Foundation of China (NFSC 31420103905, 81,430,056 and 81,321,003), the China National Major Scientific Program (973 Project-2010CB912202), and the Lam Chung Nin Foundation for Systems Biomedicine; and by grants to Caixia Ren from the National Natural Science Foundation of China (81402388); Leading Academic Discipline Project of Beijing Education Bureau

(BMU20110254) and Peking University grant (BMU20150492).

\section{Availability of data and materials}

The datasets analyzed during the current study are available from the corresponding author upon reasonable request.

\section{Authors' contributions}

$\mathrm{CR}, \mathrm{BM}$ and $\mathrm{YY}$ conceived and designed the project. YW and YS collected the samples. JL performed the LC-MS. CR, JZ and HL analyzed the data. CR, BM and YY wrote the manuscript. All authors read and approved the final manuscript.

Ethics approval and consent to participate

All subjects gave informed consent for inclusion before they participated in the study. The study was conducted in accordance with the Declaration of Helsinki, and the protocol was approved by the Ethics Committee of the Beijing University of Chinese Medicine (ECPJ-BDY-2016-06).

\section{Consent for publication}

Not applicable.

\section{Competing interests}

The authors declare that they have no competing interests.

\section{Publisher's Note}

Springer Nature remains neutral with regard to jurisdictional claims in published maps and institutional affiliations.

\section{Author details}

${ }^{1}$ Departments of Human Anatomy, Histology and Embryology, Peking University Health Science Center, Beijing 100191, China. ${ }^{2}$ Institute of Systems Biomedicine, Department of Pathology, School of Basic Medical Sciences, Peking University Health Science Center, Beijing 100191, China. ${ }^{3}$ Department of Neurology, Dongzhimen Hospital Affiliated to Beijing University of Chinese Medicine, Beijing 100700, China. ${ }^{4}$ Dongfang Hospital Affiliated to Beijing University of Chinese Medicine, Beijing 100078, China. ${ }^{5}$ Beijing Key Laboratory of Tumor Systems Biology, Peking-Tsinghua Center for Life Sciences, Beijing 100191, China.

Received: 22 September 2017 Accepted: 22 January 2018

Published online: 02 February 2018

\section{References}

1. Launer $\sqcup$, Terwindt GM, Ferrari MD. The prevalence and characteristics of migraine in a population-based cohort: the gem study. Neurology. 1999:53:537-42.

2. Headache Classification Committee of the International Headache S. The international classification of headache disorders, 3rd edition (beta version). Cephalalgia. 2013;33:629-808.

3. Gasparini CF, Smith RA, Griffiths LR. Genetic and biochemical changes of the serotonergic system in migraine pathobiology. J Headache Pain. 2017;18:20. 
4. Antonaci F, Ghiotto N, Wu S, Pucci E, Costa A. Recent advances in migraine therapy. Spring. 2016;5:637.

5. Krymchantowski AV. Acute treatment of migraine. Breaking the paradigm of monotherapy. BMC Neurol. 2004:4:4

6. Dennis EA. Lipidomics joins the omics evolution. Proc Natl Acad Sci U S A. 2009:106:2089-90.

7. Masaki Ishikawa KS, Urata M, Kumagai Y, Maekawa K, Saito Y. Comparison of circulating lipid profiles between fasting humans and three animal species used in preclinical studies: mice, rats and rabbits. Lipids Health Dis. 2015;14:104.

8. Liu J, Liu Y, Zhou J, Yin Y. Lc-ms-based lipidomic analysis of serum samples from patients with rheumatoid arthritis. Int J Clin Exp Pathol. 2017;10:2970-8.

9. Violante S, Ijlst L, Ruiter J, Koster J, van Lenthe H, Duran M, de Almeida IT, Wanders RJ, Houten SM, Ventura FV. Substrate specificity of human carnitine acetyltransferase: implications for fatty acid and branched-chain amino acid metabolism. Biochim Biophys Acta. 2013;1832:773-9.

10. Tsugawa H, Cajka T, Kind T, Ma Y, Higgins B, Ikeda K, Kanazawa M, VanderGheynst J, Fiehn O, Arita M. Ms-dial: data-independent ms/ms deconvolution for comprehensive metabolome analysis. Nat Methods. 2015; 12:523-6.

11. Xia J, Psychogios N, Young N, Wishart DS. Metaboanalyst: a web server for metabolomic data analysis and interpretation. Nucleic Acids Res. 2009;37:W652-60.

12. Ramsay RR, Gandour RD, van der Leij FR. Molecular enzymology of carnitine transfer and transport. Biochim Biophys Acta. 2001;1546:21-43.

13. Schooneman MG, Vaz FM, Houten SM, Soeters MR. Acylcarnitines: reflecting or inflicting insulin resistance? Diabetes. 2013;62:1-8.

14. Adams SH, Hoppel CL, Lok KH, Zhao L, Wong SW, Minkler PE, Hwang DH, Newman JW, Garvey WT. Plasma acylcarnitine profiles suggest incomplete long-chain fatty acid beta-oxidation and altered tricarboxylic acid cycle activity in type 2 diabetic african-american women. J Nutr. 2009;139:1073-81

15. Wang-Sattler R, Yu Z, Herder C, Messias AC, Floegel A, He Y, Heim K, Campillos M, Holzapfel C, Thorand B, Grallert H, Xu T, Bader E, Huth C, Mittelstrass K, Doring A, Meisinger C, Gieger C, Prehn C, Roemisch-Margl W, Carstensen M, Xie L, Yamanaka-Okumura H, Xing G, Ceglarek U, Thiery J, Giani G, Lickert H, Lin X, Li Y, Boeing H, Joost HG, de Angelis MH, Rathmann W, Suhre K, Prokisch H, Peters A, Meitinger T, Roden M, Wichmann HE, Pischon T, Adamski J, Illig T. Novel biomarkers for pre-diabetes identified by metabolomics. Mol Syst Biol. 2012;8:615.

16. Giesbertz P, Ecker J, Haag A, Spanier B, Daniel H. An Ic-ms/ms method to quantify acylcarnitine species including isomeric and odd-numbered forms in plasma and tissues. J Lipid Res. 2015;56:2029-39.

17. Borgman MP, Coleman T, Kolhatkar RB, Geyser-Stoops S, Line BR, Ghandehari H. Tumor-targeted hpma copolymer-(rgdfk)-(chx-a"-dtpa) conjugates show increased kidney accumulation. J Control Release 2008; 132:193-199.

18. Young SA, Mina JG, Denny PW, Smith TK. Sphingolipid and ceramide homeostasis: potential therapeutic targets. Biochem Res Int. 2012; 2012:248135

19. Jana A, Hogan EL, Pahan K. Ceramide and neurodegeneration: susceptibility of neurons and oligodendrocytes to cell damage and death. J Neurol Sci. 2009;278:5-15.

20. Maja Jazvinšćak Jembrek PRH, Goran Šimić. Ceramides in alzheimer's disease: Key mediators of neuronal apoptosis induced by oxidative stress and a $\beta$ accumulation. Oxid Med Cell Longev. 2015;2015:346783. Published online 2015 May 24. https://doi.org/10.1155/2015/346783PMCID:PMC4458271.

21. Nikolova-Karakashian MN, Rozenova KA. Ceramide in stress response. Adv Exp Med Biol. 2010;688:86-108.

22. de la Monte SM. Triangulated mal-signaling in alzheimer's disease: roles of neurotoxic ceramides, er stress, and insulin resistance reviewed. J Alzheimers Dis. 2012;30(Suppl 2):S231-49.

23. Mielke MM, Haughey NJ, Bandaru W, Schech S, Carrick R, Carlson MC, Mor S, Miller MI, Ceritoglu C, Brown T, Albert M, Lyketsos CG. Plasma ceramides are altered in mild cognitive impairment and predict cognitive decline and hippocampal volume loss. Alzheimers Dement. 2010;6:378-85.

24. Vanmolkot FH, de Hoon JN. Increased c-reactive protein in young adult patients with migraine. Cephalalgia. 2007;27:843-6.

25. Cha MH JA, Ko MM, Zhang C, Lee MS. Metabolic profiles distinguish nondampness-phlegm and dampness-phlegm patterns among korean patients with acute cerebral infarction. Evid Based Complement Alternat Med. 2013; 2013:517018. https://doi.org/10.1155/2013/517018.
26. Matsumoto T, Kobayashi T, Kamata K. Role of lysophosphatidylcholine (lpc) in atherosclerosis. Curr Med Chem. 2007;14:3209-20.

27. Calzada E, Onguka O, Claypool SM. Phosphatidylethanolamine metabolism in health and disease. Int Rev Cell Mol Biol. 2016;321:29-88.

28. Geyik S, Altunisik E, Neyal AM, Taysi S. Oxidative stress and DNA damage in patients with migraine. J Headache Pain. 2016;17:10.

29. Tuncel D, Tolun Fl, Gokce M, Imrek S, Ekerbiçer H. Oxidative stress in migraine with and without aura. Biol Trace Elem Res. 2008;126:92-7.

30. Eren Y, Dirik E, Neselioglu S, Erel O. Oxidative stress and decreased thiol level in patients with migraine: cross-sectional study. Acta Neurol Belg. 2015;115:643-9.

31. Alp R, Selek S, Alp SI, Taskin A, Kocyigit A. Oxidative and antioxidative balance in patients of migraine. Eur Rev Med Pharmacol Sci. 2010;14:877-82.

32. Carri MT, Valle C, Bozzo F, Cozzolino M. Oxidative stress and mitochondrial damage: importance in non-sod1 als. Front Cell Neurosci. 2015;9:41.

33. Lau SK, Lee KC, Lo GC, Ding VS, Chow WN, Ke TY, Curreem SO, To KK, Ho DT, Sridhar S, Wong SC, Chan JF, Hung IF, Sze KH, Lam CW, Yuen KY, Woo PC. Metabolomic profiling of plasma from melioidosis patients using uhplcqtof ms reveals novel biomarkers for diagnosis. Int J Mol Sci. 2016;17:307.

34. Park SJ, Lee KP, Im DS. Action and signaling of lysophosphatidylethanolamine in mda-mb-231 breast cancer cells. Biomol Ther (Seoul). 2014:22:129-35.

35. Park KS, Lee HY, Lee SY, Kim MK, Kim SD, Kim JM, Yun J, Im DS, Bae YS Lysophosphatidylethanolamine stimulates chemotactic migration and cellular invasion in sk-ov3 human ovarian cancer cells: involvement of pertussis toxinsensitive g-protein coupled receptor. FEBS Lett. 2007:581:4411-6.

36. Lee JM, Park SJ, Im DS. Calcium signaling of lysophosphatidylethanolamine through Ipa1 in human sh-sy5y neuroblastoma cells. Biomol Ther (Seoul). 2017; 25:194-201.

37. Eikermann-Haerter K, Arbel-Ornath M, Yalcin N, Yu ES, Kuchibhotla KV, Yuzawa I, Hudry E, Willard CR, Climov M, Keles F, Belcher AM, Sengul B, Negro A, Rosen IA, Arreguin A, Ferrari MD, van den Maagdenberg AM, Bacskai BJ, Ayata C. Abnormal synaptic ca(2+) homeostasis and morphology in cortical neurons of familial hemiplegic migraine type 1 mutant mice. Ann Neurol. 2015:78:193-210.

\section{Submit your next manuscript to BioMed Central and we will help you at every step:}

- We accept pre-submission inquiries

- Our selector tool helps you to find the most relevant journal

- We provide round the clock customer support

- Convenient online submission

- Thorough peer review

- Inclusion in PubMed and all major indexing services

- Maximum visibility for your research

Submit your manuscript at www.biomedcentral.com/submit
) Biomed Central 